INPLASY

PROTOCOL

To cite: Almulihi et al.

Comparison of safety and effectiveness between levetiracetam and phenytoin in treatment Pediatric Status Epilepticus: A meta-analysis. Inplasy protocol 2021100082. doi:

10.37766/inplasy2021.10.0082

Received: 21 October 2021

Published: 21 October 2021

Corresponding author:

Qasem Almulihi

qasem.almulihi@hotmail.com

Author Affiliation:

King Fahad University Hospital

- Saudi Arabia.

Support: No.

Review Stage at time of this submission: Data extraction Completed but not published.

Conflicts of interest:

None declared.

\section{Comparison of safety and effectiveness between levetiracetam and phenytoin in treatment Pediatric Status Epilepticus: A meta-analysis}

Almulihi, Q1'; Almuhanna, F2; Alsultan, E³ Almuhanna, M.

Review question / Objective: To evaluate the safety and effectiveness of levetiracetam and phenytoin by evaluating the events of seizure termination and recurrence in children. Condition being studied: Convulsive Status epilepticus (CSE) is a serious neurological emergency in children that requires prompt treatment and termination to obtain satisfactory outcomes that control potential neurological consequences, which increase mortality and morbidity.

Information sources: We used the internet databases PubMed, Embase, and Google Scholar to conduct a literature search for the appropriate studies. A meta-analysis was performed to calculate the odds ratio using fixed and randomeffects models.

INPLASY registration number: This protocol was registered with the International Platform of Registered Systematic Review and Meta-Analysis Protocols (INPLASY) on 21 October 2021 and was last updated on 21 October 2021 (registration number INPLASY2021100082).

\section{INTRODUCTION}

Review question / Objective: To evaluate the safety and effectiveness of levetiracetam and phenytoin by evaluating the events of seizure termination and recurrence in children.
Condition being studied: Convulsive Status epilepticus (CSE) is a serious neurological emergency in children that requires prompt treatment and termination to obtain satisfactory outcomes that control 
potential neurological consequences, which increase mortality and morbidity.

\section{METHODS}

Search strategy: The search was performed according to the guidelines recommended by the PRISMA statement for systematic reviews and meta-analyses. Participants, interventions, comparison, and outcomes (PICO) annotations were used to determine the parameters and annotate the data. Literature was searched for the appropriate studies from the online databases of PubMed, Embase, and Google Scholar published up to August 2021. The key words used for the search were as follows: "status epilepticus", "seizure", "levetiracetam", "phenytoin", "pediatric", and "children".

Participant or population: Pediatric Status Epilepticus.

Intervention: Seizure termination and seizure recurrence.

Comparator: Levetiracetam vs. phenytoin.

Study designs to be included: All studies were reviewed by two reviewers. According to the eligibility criteria, the papers were evaluated and screened for data relevance. We included all studies that compared the effectiveness of levetiracetam and phenytoin by the events of seizure termination and recurrence in children.

Eligibility criteria: We included all studies that compared the effectiveness of levetiracetam and phenytoin by the events of seizure termination and recurrence in children.

Information sources: We used the internet databases PubMed, Embase, and Google Scholar to conduct a literature search for the appropriate studies. A meta-analysis was performed to calculate the odds ratio using fixed and random-effects models.

Main outcome(s): Events of seizure termination and recurrence in children.
Quality assessment / Risk of bias analysis: One investigator independently extracted the data and input them into Microsoft Excel 2020. The events of seizure termination and recurrence for levetiracetam and phenytoin were extracted for the meta-analysis. Cochrane risk-of-bias tool for randomized trials version 2 (RoB-2). was used to assess quality. One independent reviewer assessed all domains including bias arising from the randomization process, bias due to deviations from intended interventions, bias due to missing outcome data, bias in measurement of the outcome, and bias in selection of the reported results. Each risk of bias point was rated as either "low", "some concerns", or "high" risk of bias.

Strategy of data synthesis: RevMan (5.4; The Cochrane Collaboration) was used to perform all statistical analyses. In forest plots, we used the Mantel-Haenszel (M-H) method to analyze the dichotomous data (events/total), and the odds ratio was used to determine strength of their association. Heterogeneity (12), which is the variation in study outcomes, was calculated using the Higgins 12 statistic and Chi-squared ( $x 2)$ tests. Based on the heterogeneity value, either fixed or random effect model was used to calculate the effect size of the study. For heterogeneity of $>50 \%$ or $P \leq$ 0.05 , the random-effect model was used for the otherwise fixed effect model. Higher heterogeneity corresponds to higher variation in studies. $A Z$ score $\geq 1.96$ or $p$ value $\leq 0.05(Z \geq 1.96$ or $P \leq 0.05)$ are considered significant.

\section{Subgroup analysis: No.}

Sensitivity analysis: We analyzed the efficacy seizure termination within $24 \mathrm{~h}$ of study drug infusion. The incidences of seizure termination within $24 \mathrm{~h}$ were $76.9 \%$ $(1078 / 1401)$ for levetiracetam and $70.5 \%$ $(919 / 1303)$ for phenytoin. Levetiracetam had a significantly higher number of seizure termination events than phenytoin $(P=0.005,12=66 \%)$ [Figure 2].The incidences of seizure recurrence within 24 $h$ were $10 \%(76 / 760)$ for levetiracetam and $15.6 \%(105 / 670)$ for phenytoin. Phenytoin 
had a significantly higher number of seizure recurrence events than levetiracetam $(P=0.005,12=66 \%)$.

Language: English.

Country(ies) involved: Saudi Arabia.

Keywords: The key words used for the search were as follows: "status epilepticus", "seizure", "levetiracetam", "phenytoin", "pediatric", and "children".

Contributions of each author:

Author 1 - QASEM ALMULIHI.

Author 2 - FATIMAH ALMUHANNA.

Author 3 - EMAN ALSULTAN.

Author 4 - MOHAMED ALMUHANNA. 